\title{
Acknowledgement to Reviewers of Coatings in 2015
}

\author{
Coatings Editorial Office, \\ Published: 22 January 2016 \\ MDPI AG, Klybeckstrasse 64, CH-4057 Basel, Switzerland; coatings@mdpi.com
}

The editors of Coatings would like to express their sincere gratitude to the following reviewers for assessing manuscripts in 2015.

We greatly appreciate the contribution of expert reviewers, which is crucial to the journal's editorial decision-making process. Several steps have been taken in 2015 to thank and acknowledge reviewers. Good, timely reviews are rewarded with a discount off their next MDPI publication. By creating an account on the submission system, reviewers can access details of their past reviews, see the comments of other reviewers, and download a letter of acknowledgement for their records. This is all done, of course, within the constraints of reviewer confidentiality. Feedback from reviewers shows that most see their task as a voluntary and mostly unseen work in service to the scientific community. We are grateful to our reviewers for the contribution they make.

Abdul-Aziz, Ali
Abdulgader, M.
Ahn, Byung Tae
Akhavan, Omid
Alipour, Y.
Almond, Darryl P.
Álvarez, José Ignacio
Amadelli, R.
Antunes, Maria Dulce
Anzai, Jun-ichi
Aranguren, Mirta I.
Ares, A.E.
Bae, Byeong-Soo
Bahamonde, A.
Barringer, S.
Barron, Andrew R.
Belgacem, Mohamed Naceur
Beyrau, Frank
Blayo, Anne
Bolelli, Giovanni
Bonetta, Silvia
Bonora, Pierluigi
Burrows, Susan
Calnan, Sonya
Caporali, Stefano
Casalegno, Mose
Charitidis, Costas A.
Chen, Hsien-Yeh
Cheng, Kong-Wei
Cirvilleri, Gabriella

Huang, E-Wen
Ilavsky, Jan
Innocenti, Massimo
Ionescu, Mihail
Jabbarzadeh, Ahmad
Jacobs, Karin
Jérôme, Faure-Vincent
Keller, T.
Kemnitz, Erhard
Kichambare, PadmakarD.
Kim, Choong-Un
Kim, Hak Yong
Kim, Yoong Ahm
Kiwi, John
Klotz, Ulrich E.
Kranz, L.
Kraus, Tobias
Kuei, Chan Taw
Kulinich, S.A.
Kumar, S.
Lahann, Joerg
Landry, Veronic
Lange, Heiko
Lavorgna, Marino
Lee, Thomas
Lettieri, Mariateresa
Li, Sunny R.
Lin, Tong
Liu, Yingchi
Loeber, T.H.

\author{
Pasbakhsh, Pooria \\ Paul, S. \\ Pavia, Sara \\ Pearton, S.J. \\ Pedersen, Henrik \\ Peng, Yucheng \\ Philipp, Claudia \\ Piergiovanni, Luciano \\ Planche, Marie-pierre \\ Pojman, John A. \\ Pommer, Bernhard \\ Poon, Chi-Sun \\ Pretsch, Thorsten \\ Puglisi, Rosaria \\ Pulpytel, Jerome \\ Quan, Taihao \\ Ramirez, Anibal Maury \\ Randazzo, Walter \\ Rapino, Stefania \\ Raucci, M.G. \\ Razza, Stefano \\ Reale, A. \\ Refait, Ph. \\ Riikonen, Juha \\ Rizza, Giancarlo \\ Robinson, Sara C. \\ Romanyuk, YE \\ Sa'ncheza, Luis \\ Saha, Gobinda \\ Saito, Kozo
}




Cocetta, Giacomo
Cojocaru-Mirédin, Oana
Corcione, C. Esposito
Costa, Rui
Criado Sanz, Maria
D'Aquino, Salvatore
De Cogan, Felicity
Dou, Maofeng
Drouiche, Nadjib
Duan, Fei
Dumitrica, Traian
Ellinas, Kosmas
Erich, S.J.F.
Evans, Drew
Faber, Hendrik
Faisal, N.H.
Falco, Aniello
Farris, Stefano
Felner, Israel
Figueira, Rita
Forsyth, Maria
Frigione, Mariaenrica
Fürbeth, Wolfram
Galca, A.C.
Garrigós, M.C.
Gelderblom, Hanneke
Gervasini, Antonella
Gupta, Surojit
Hall, Colin
Hayakawa, Tohru
Heidary, Damoon S. Baba
Hippler, Rainer
Holmberg, Kenneth

\author{
Lynn, Brittany \\ Mahmoudi, Seyed \\ Mai, C. \\ Maireles Torres, Pedro J. \\ Major, Jonathan D. \\ Malucelli, Giulio \\ Manthina, Venkata \\ Mariniello, Loredana \\ Marrocchi, Assunta \\ Martinez, Thomas \\ McNamara, Bruce \\ Méndez-Ardoy, A. \\ Meola, Carosena \\ Miotello, Antonio \\ Montgomery, M. \\ Moseke, Claus \\ Mosquera, Maria \\ Munz, Richard J. \\ Nawani, Pranav \\ Nejad, Mojgan \\ Ngo, Truc T. \\ Nolte, Adam J. \\ Öhlund, Thomas \\ Oksa, Maria \\ Ortelli, Simona \\ Palmisano, Leonardo \\ Park, Nokeun \\ Parnell, Andrew J. \\ Tait, Jeffrey G. \\ Tanaka, Kunihiko \\ Teisala, Hannu \\ Tobaldi, D.M. \\ Truss, Rowan
}

\author{
Saito, Yasuhiro \\ Salimi Jazi, Mehdi \\ Santodonato, Lou \\ Schlegel, N. \\ Seiler, Philipp \\ Seok, Chang-Sung \\ Shimizu, Kazuo \\ Shimoi, Norihiro \\ Silva, F.J.G. \\ Silvestre, Clara \\ Sovak, Guy \\ Stober, L \\ Sun, Keju \\ Tsay, Chien-Yie \\ Tshabalala, Mandla A. \\ Varshney, Vikas \\ Vartiainen, Jari \\ Verbeek, J.R. \\ Vivo, Paola \\ Wang, Zuankai \\ Wang, Yuxin \\ Wang, Qinyi \\ Ward, L.P. \\ Welle, Alexander \\ Wilden, Johannes \\ Witte, Wolfram \\ Xiao, Xudong \\ Yeo, Boon Siang \\ $\mathrm{Yu}$, Chang-Feng \\ Zakutayev, Andriy \\ Zhang, Qiang \\ Zhao, Lidong \\ Zharnikov, Michael
}

(C) 2016 by the author; licensee MDPI, Basel, Switzerland. This article is an open access article distributed under the terms and conditions of the Creative Commons by Attribution (CC-BY) license (http://creativecommons.org/licenses/by/4.0/). 